\title{
Biaxial Extensometer for Measuring of the Human Skin Anisotropy in Vivo
}

\author{
L. Capek, Z. Lochman., L. Dzan and E. Jacquet
}

\begin{abstract}
Knowing biomechanical properties of human skin is important for clinical decision making as well as clinical intervention. Measuring these properties in vivo is essential. However, existing devices don't respect the fact, that human skin is an anisotropic material. Nowadays devices investigate mostly the human skin anisotropy by uniaxial testing. This leads leads to distorted information. In this paper we proposed a new biaxial device for testing the human skin in vivo. This device can be used to obtain the true anisotropy of human skin.
\end{abstract}

\section{INTRODUCTION}

$\mathrm{R}$ ESEARCH into the biomechanics of the human skin has attracted a lot attention among dermatologists. Skin performs a wide variety of functions resulting from chemical and physical reactions inside its components. The major function of skin is to act as a barrier to the exterior environment. From the mechanical point of view human skin is a heterogeneous material composed of collagen and elastin fibres in a proteoglycan matrix [1]. Acting together, these components are responsible for the complex mechanical behaviour of skin as manifested in stress, strain curves. These curves are characterized by a low-stiffness region at small strains followed by a dramatic increase in stiffness as the strain becomes large [2], [3]. Moreover, its behaviour depends on direction.

Wijn et al. conducted uniaxial experiments on the leg in parallel and perpendicular directions to Langer's lines and discovered that the Young's moduli was 3.8 times greater in parallel direction [4]. Khatyr et al. showed that the anisotropy ratio on the forearm was equal to 5 [5]. Manschot et al. showed that on the leg $76 \%$ of elastic fibres were oriented along Langer's lines and only $5.1 \%$ were oriented perpendicularly [6].

There are several non-invasive and invasive techniques for the evaluation of mechanical properties of human skin. The most commonly used methods are based on the measurement of suction, torsion and traction [3], [7], [8],

Manuscript received August 10, 2010. This work was supported in part by the Regional hospital of Liberec under Grant VR 090303

L. Capek, Department of Applied Mechanics, Technical University of Liberec, Czech Republic (e-mail:lukas.capek@tul.cz) - corresponding author.

Z. Lochman, Department of Applied Mechanics, Technical University of Liberec, Czech Republic (e-mail: zdenek.lochman@tul.cz)

L. Dzan Department of Maxillo-Facial Surgery, Regional Hospital of Liberec, Czech Republic (e-mail: ladislav.dzan@nemlib.cz).

E. Jacquet, FEMTO-ST, University of Franche-Comte, France (e-mail: emmanuelle.jacquet@univ-fcomte.fr)
[9], [10]. Other experimental methods used to measure the mechanical behavior of the skin elastic wave propagation and indentation [11]. However, the data obtained by these methods are mainly descriptive and often very different, depending on the experimental conditions. The most common test is the uniaxial one. It works by applying displacements to the skin using two extensible pads that are attached to the skin. The force needed for deformation is measured by a load cell as the skin deforms.

The aim of this study was to construct a prototype of a biaxial extensometer allowing the measurement of the directionally dependence of biomechanical properties of human skin in vivo.

\section{MAterial AND Methods}

In spite of the fact that there are lot of uniaxial in vivo extensometers, there is no device, to our knowledge, enabling to load the human skin in two directions simultaneously in vivo. The design of the extensometer was done in the CAD software Pro/Engineer (PTC software ltd., Czech Republic).

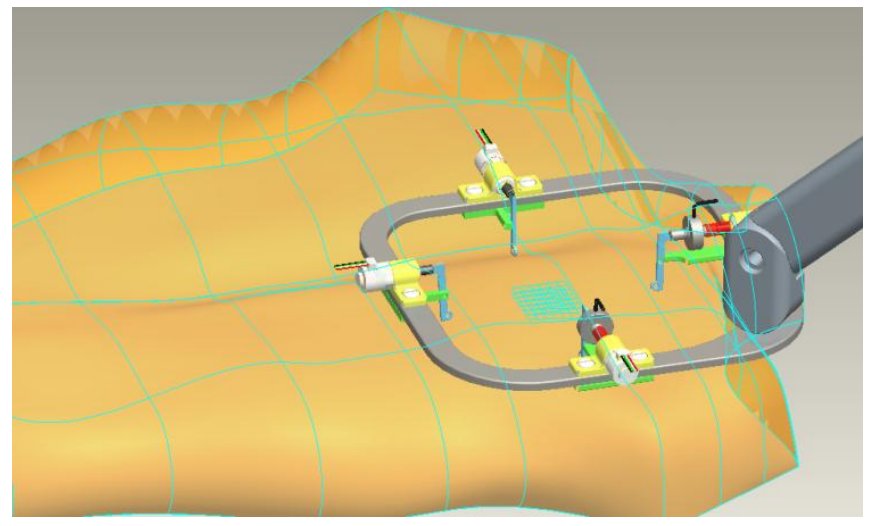

Fig. 1. The CAD model of the biaxial system.

The device consists of two miniature load cells, four actuators, fours pads and a movable frame, Fig. 1.

The input parameters: low mass, high durability, variability and low cost. For optimizing of these parameters an finite element analysis was performed. The movement of two opposite actuators is tied with maximal speed of 600 steps/second and maximal displacement of $26 \mathrm{~mm}$. The movement from the actuators to pads is transfered by a lever system. The pads are joined to the skin surface by doubled 
sided adhesive tape. The distance between two opposite pads is adjustable, but the minimum distance is $30 \mathrm{~mm}$. The final design of the real biaxial extensometer can be seen on the Fig. 2. All components were made from duralumin, which meets the low mass with high durability criteria.

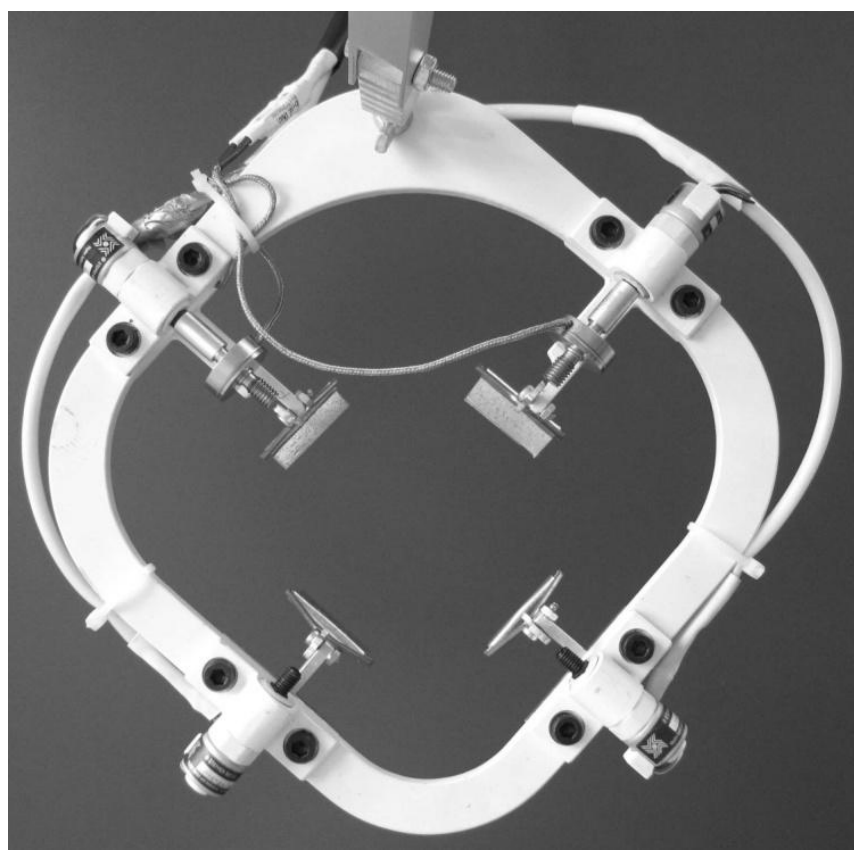

Fig. 2. The real biaxial system.

We have conducted three main test to validate our new biaxial extensometer. All of them were performed at the region of human forearm:

- The first one was done in axis $\mathrm{x}$ (parallel to Langer's lines),

- the second one in y axis (perpendicular to Langer's lines)

- the last one in $\mathrm{x}$ and $\mathrm{y}$ axis together.

\section{RESULTS}

All gained stress and time curves exhibit the initial, lowstiffness region, where the collagen fibres align themselves parallel to the maximum stretch direction while either the elastin and/or the proteoglycan matrix provides resistance to deformation. Once the collagen fibres are aligned, further extension of the skin requires extension of the collagen fibbers, resulting in a significant increase in skin stiffness until failure.

The typical curves are presented below. In the first test carried out in $\mathrm{x}$ direction the maximum recorded force was $2.45 \mathrm{~N}$, Fig. 3. In the second test carried out in y direction the maximum recorded force was $6.5 \mathrm{~N}$, Fig. 4. In the last test the maximum recorded force in $\mathrm{x}$ direction was $6.6 \mathrm{~N}$ and in perpendicular direction 7.5 N, Fig. 5 .

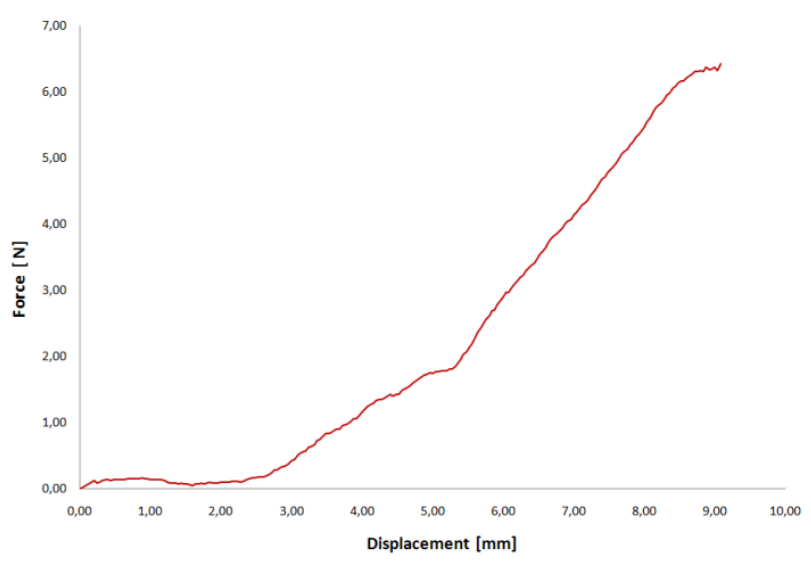

Fig. 3. The force displacement curve from uniaxial in vivo test along the Langer's lines on the forearm.

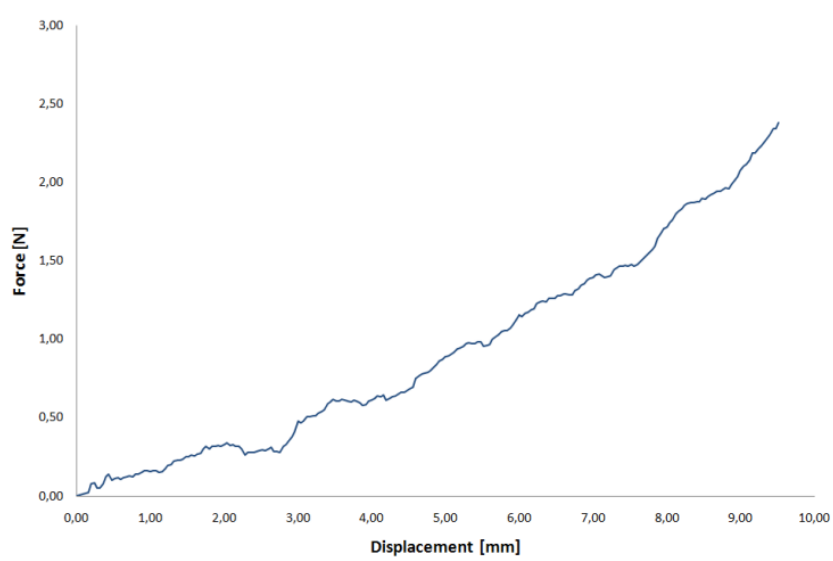

Fig. 4. The force displacement curve from uniaxial in vivo test perpendicular to the Langer's lines on the forearm.

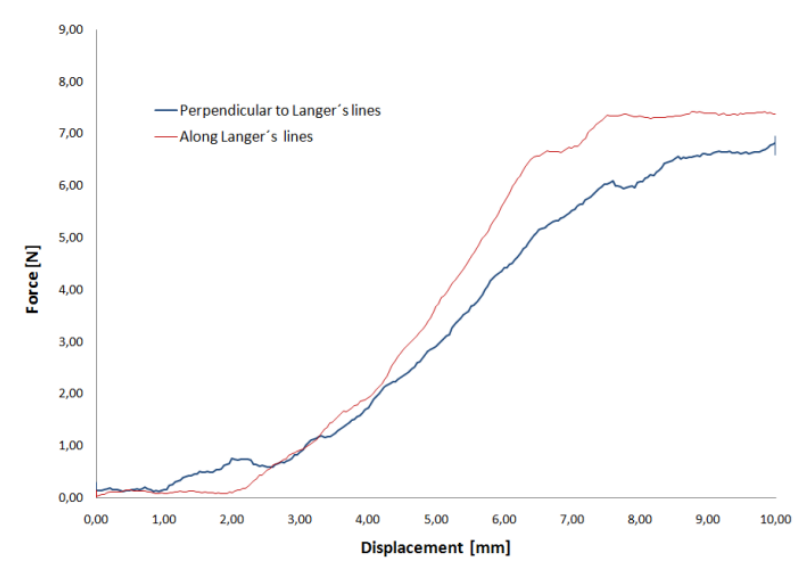

Fig. 5. The force displacement curve from biaxial in vivo test on the forearm. 


\section{CONCLUSIONS}

We assume that the tensile tests are, from a mechanical point of view, one of the best possible load applications to identify skin anisotropy. Human skin exhibits nonlinear anisotropic properties. To identify human skin anisotropy biaxial tests must be performed. Nowadays the most common tests used to identify human skin anisotropy are uniaxial tests, which are done separately in different directions. From the mechanical point of view this doesn't provide us with all the information about human skin anisotropy. According to literature, there is no biaxial device enabling tensile tests on human skin in vivo.

The proposed biaxial device is the first prototype of its kind. All gained stress and time curves exhibit the nonlinear behaviour with values close known from the literature [2], [3]. The most important data can be seen Fig. 6, where two stress strain curves were gained in the same time. Human skin anisotropy is well-known property of the human skin. Nevertheless, if on looks at the literature in the field it has not received much attention. It has mostly been dealt with within the context of uniaxial tests.

It should be noted, that only preliminary tests were done and results are not valid from the statistical point of view. In the future the results will be confronted by a correlation system Dantec Q-400 (Dantec Dynamics LtD., Germany) and the statistical results must be gained. The aim of this paper was to present our new biaxial system constructed for measuring human skin in vivo.

\section{ACKNOWLEDGMENTS}

We would like to thank Dr. Aleš Lufinka for technical help during this project.

\section{REFERENCES}

[1] C. Robinson, W. Hanke, R. Sengelimann, D. Siegel, Surgery of the Skin: Procedural Dermatology, Portland: Elsevier Mosby, 2005, ch. 2.

[2] Y.C. Fung, Biomechanics, New York: Springer, 1993, pp. 302-306.

[3] Agache P., P. Humbert, H. Maibach, Measuring the skin. Heidelberg: Springer, 2004.

[4] PFF. Wijn, Ajm. Brakee, J.P. Kuiper, A. Vendrik, The alinear viscoelastic properties of the human skin in vivo related to sex and age, Lancaster: Marks and Payne PA Editions Bioengineering and the skin MTP Press, 1981, pp. 135-146.

[5] F. Khatyr, C. Imberdis, P. Vescovo, D. Varchon, J.M. Lagarde, "Model of the viscoelastic behaviour of skin in vivo and study of anisotropy", Skin research and technology, vol.10, pp. 96-103, 2004.

[6] B. Manschotte, "Measurement and modeling of the mechanical properties of the human skin in vivo", Journal de biomechanics, vol. 19, pp. 511-515, 1986.

[7] J.H. Evans, W. Siesennop, "Controlled quasi-static testing of human skin in vivo", presented at Digest of the Seventh International Conference on Medical and Biological Engineering, Stockholm, 1967.

[8] C.W. Gunner, W. C.Hutton, T. E.Burlin, "The mechanical properties of skin in vivo - a portable hand held extensometer", British Journal of Dermatology, vol. 100, pp. 161-163, 1979.

[9] P. Vescovo, P., ,Validation of measuring technique for identifying of Young's moduli of human skin“, $\mathrm{PhD}$ dissertation, Universite' de Franche-Comte, 2002.

[10] K.H. Lim et al., „New extensometer to measure in vivo uniaxial mechanical properties of human skin", Journal of Biomechanics, vol. 41, pp. 931-936, 2008.

[11] C. Pailler-Matte'i, H. Zahouani, "Analysis of adhesive behaviour of human skin in vivo by an indentation test", Tribology Int., vol. 39, pp $12-21,2006$ 